\title{
Selênio eritrocitário e sua relação com o perfil lipídico em mulheres obesas
}

\author{
Erythrocyte selenium and its relationship with the lipid profile in obese women \\ Eritrocitos selenio y su relación con el perfil lípido en mujeres obesas
}

Recebido: 02/03/2021 | Revisado: 07/03/2021 | Aceito: 10/03/2021 | Publicado: 19/03/2021

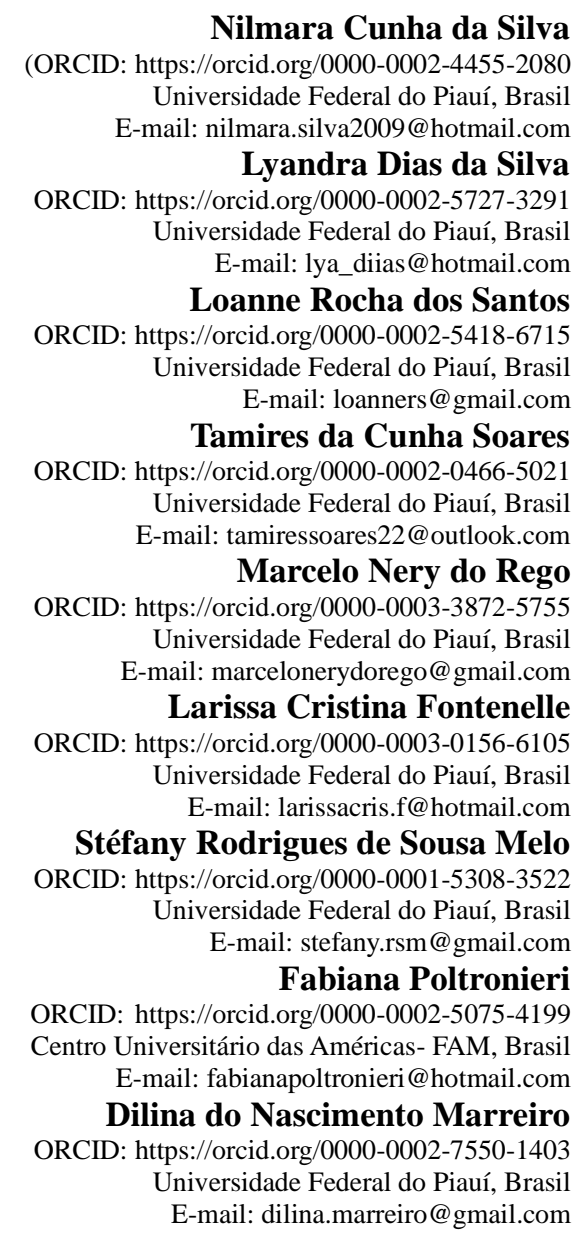

\begin{abstract}
Resumo
O presente estudo teve como objetivo identificar a existência da relação entre parâmetros do selênio dietético e eritrocitário e marcadores do perfil lipídico em mulheres com obesidade. Trata-se de um estudo caso- controle envolvendo 56 mulheres, com idade entre 20 a 50 anos, sendo distribuídas em dois grupos: grupo caso (obesas, $\mathrm{n}=22$ ) e grupo controle (eutróficas, $n=34$ ). Foram avaliadas as concentrações de selênio dietético e eritrocitário e perfil lipídico (colesterol total, triacilgliceróis, HDL-colesterol, LDL-colesterol e VLDL-colesterol). O projeto foi aprovado pelo Comitê de Ética em Pesquisa da Universidade Federal do Piaú, sob número de parecer 2.014.100. Os dados foram analisados por meio do programa estatístico SPSS for Windows 20.0 ® ${ }^{\circledR}$. Quanto aos resultados, observou-se que os valores médios da ingestão dietética de selênio estavam adequados de acordo com as recomendações da ingestão diária recomendada de $55 \mu \mathrm{g}$ de selênio $(\mathrm{p}>0,05)$. As mulheres obesas apresentaram concentrações eritrocitárias de selênio reduzidas quando comparadas ao grupo controle $(\mathrm{p}<0,05)$. Sobre o perfil lipídico, as mulheres obesas apresentaram parâmetros elevados de colesterol total, triacilgliceróis, LDL-c e VLDL-c e valores reduzidos de HDL-c, quando comparados ao grupo controle $(\mathrm{p}<0,05)$. Além disso, não se observou correlação significativa entre o selênio dietético e eritrocitário e os parâmetros do perfil lipídico entres os grupos avaliados. Diante dos resultados, o estudo não revela correlação entre o marcador dietético e eritrocitário de selênio e os parâmetros do perfil lipídico.
\end{abstract}

Palavras-chave: Selênio; Dislipidemia; Obesidade. 


\begin{abstract}
The present study aimed to identify the relationship between parameters of dietary and erythrocyte selenium and lipid profile markers in women with obesity. This is a case-control study involving 56 women, aged between 20 and 50 years, divided into two groups: case group (obese, $\mathrm{n}=22$ ) and control group (eutrophic, $\mathrm{n}=34$ ). The concentrations of dietary and erythrocyte selenium and lipid profile (total cholesterol, triacylglycerols, HDL-cholesterol, LDLcholesterol and VLDL-cholesterol) were evaluated. The project was approved by the Research Ethics Committee of the Federal University of Piauí, under opinion number 2,014,100. The data were analyzed using the statistical program SPSS for Windows 20.0 ®. As for the results, it was observed that the average values of dietary selenium intake were adequate according to the recommendations of the recommended daily intake of $55 \mu \mathrm{g}$ of selenium (p> 0.05 ). Obese women had reduced erythrocyte concentrations of selenium when compared to the control group ( $\mathrm{p}$ $<0.05)$. Regarding the lipid profile, obese women showed high parameters of total cholesterol, triglycerides, LDL-c and VLDL-c and reduced HDL-c values, when compared to the control group ( $\mathrm{p}<0.05)$. In addition, there was no significant correlation between dietary and erythrocyte selenium and the parameters of the lipid profile between the groups evaluated. In view of the results, the study reveals no correlation between the dietary and erythrocyte selenium marker and the parameters of the lipid profile.
\end{abstract}

Keywords: Selenium; Dyslipidemia; Obesity.

\title{
Resumen
}

El presente estudio tuvo como objetivo identificar la relación entre los parámetros del selênio dietético y eritrocitario y los marcadores del perfil lipídico en mujeres con obesidad. Se trata de un estudio de casos y controles en el que participaron 56 mujeres, con edades comprendidas entre 20 y 50 años, divididas en dos grupos: grupo de casos (obesidad, $\mathrm{n}=22$ ) y grupo de control (eutróficas, $\mathrm{n}=34$ ). Se evaluaron las concentraciones de selenio dietético y eritrocitario y el perfil lipídico (colesterol total, triacilgliceroles, colesterol HDL, colesterol LDL y colesterol VLDL). El proyecto fue aprobado por el Comité de Ética en Investigación de la Universidad Federal de Piauí, bajo la opinión número 2.014.100. Los datos se analizaron mediante el programa estadístico SPSS para Windows 20.0 ®. En cuanto a los resultados, se observó que los valores promedio de ingesta dietética de selenio fueron adecuados según las recomendaciones de la ingesta diaria recomendada de $55 \mu \mathrm{g}$ de selenio ( $>>0.05$ ). Las mujeres obesas tenían concentraciones reducidas de selenio en eritrocitos en comparación con el grupo de control ( $p<0,05)$. En cuanto al perfil lipídico, las mujeres obesas mostraron altos parámetros de colesterol total, triglicéridos, LDL-c y VLDL-c y valores reducidos de HDL-c, en comparación con el grupo control ( $p<0,05)$. Además, no hubo correlación significativa entre el selenio dietético y eritrocitario y los parámetros del perfil lipídico entre los grupos evaluados. A la vista de los resultados, el estudio no revela correlación entre el marcador de selenio dietético y eritrocitario y los parámetros del perfil lipídico.

Palabras clave: Selenio; Dislipidemia; Obesidad.

\section{Introdução}

A obesidade é uma doença crônica caracterizada pelo acúmulo excessivo de tecido adiposo, resultante do balanço energético positivo e de interações multifatoriais. A expansão do tecido adiposo favorece a sua disfunção, promovendo a secreção alterada de adipocinas, responsável por diversas alterações metabólicas tais como: estresse oxidativo, resistência à insulina, inflamação crônica de baixo grau e dislipidemia (Choe et al.,2016; Fontenelle et al., 2018).

Destaca-se que a dislipidemia é caracterizada por parâmetros do perfil lipídico alterados, como concentrações plasmáticas elevadas de colesterol total, triacilgliceróis e lipoproteínas de baixa densidade (LDL-c), bem como concentrações reduzidas de lipoproteínas de alta densidade (HDL-c) (Xavier et al., 2013; Tangvarasittichai, 2015; Vekic et al., 2018; Uranga \& Keller, 2019).

Evidências científicas apontam a participação de alguns micronutrientes no metabolismo dos lipídios, a exemplo do selênio. Esse mineral desempenha papel importante contra o estresse oxidativo, regulando a modificação oxidativa dos lipídios. Além disso, o nutriente está envolvido no aumento da atividade do receptor de LDL-c e diminuição da expressão de 3hidroxi-3-metilglutaril coenzima A redutase (HMG-CoA redutase), favorecendo redução nas concentrações de colesterol total, LDL-c e apoliproteínas B (Dhingra \& Bansal, 2006; Bleys et al., 2008; Yang et al., 2010; Su et al., 2015).

Nesse sentido, estudos mostram a existência de relação entre os valores plasmáticos de selênio e marcadores do perfil lipídico, a exemplo da pesquisa realizada por Ju et al. (2017) que identificaram em indivíduos com concentrações séricas reduzidas de selênio, maior risco de dislipidemias. De forma semelhante, os estudos conduzidos por Stranges et al. (2010) e 
Fülöp et al. (2013) também encontraram associação inversa entre as concentrações de selênio e lipídios séricos.

No entanto, estudos recentes têm evidenciado comprometimento do metabolismo do selênio em indivíduos obesos, caracterizado por reduzidos valores plasmáticos e eritrocitários decorrentes da ingestão inadequada desse nutriente. Essa condição pode comprometer a ação de selenoproteínas, e consequentemente, suas funções fisiológicas no organismo, favorecendo a susceptibilidade dos tecidos a danos oxidativos (Alasfar et al., 2011; Petry et al., 2013; Fontenelle et al., 2018).

Considerando a importância da disfunção do tecido adiposo presente em indivíduos obesos como fator contribuinte para manifestação de dislipidemias, bem como a participação do selênio no metabolismo dos lipídios, o presente estudo foi conduzido na perspectiva de identificar a existência da relação entre parâmetros do selênio e marcadores do perfil lipídico em mulheres com obesidade.

\section{Metodologia}

\subsection{Caracterização do Estudo e Protocolo Experimental}

Estudo transversal envolvendo 56 mulheres na faixa etária de 20 a 50 anos, que foram distribuídas em dois grupos: grupo caso $(n=22)$ e grupo controle $(n=34)$. A definição da amostra do estudo foi baseada na amostragem por conveniência, sendo que as participantes obesas foram recrutadas a partir da demanda espontânea de ambulatórios clínicos da cidade de Teresina. A pesquisa trata de uma investigação de natureza quantitativa, conforme Pereira et al. (2018).

Como as participantes foram selecionadas por meio de entrevista, os seguintes critérios de inclusão foram considerados: índice de massa corporal entre 18,5 e $24,9 \mathrm{~kg} / \mathrm{m}^{2}$ (grupo controle) ou entre 30 e $39,9 \mathrm{~kg} / \mathrm{m}^{2}$ (grupo obeso); não fumantes; sem diabetes mellitus, insuficiência renal crônica, doenças hepáticas, doenças cardiovasculares; não lactantes; não fazer uso de medicamentos e/ou suplementos nutricionais que possam interferir no estado nutricional relativo ao selênio e nos distúrbios do metabolismo lipídico.

O projeto foi aprovado pelo Comitê de Ética em Pesquisa da Universidade Federal do Piaú, com o número de parecer 2.014.100, como prevê a Resolução 466/12 do Conselho Nacional de Saúde (CNS) (Brasil, 2012).

As participantes assinaram o Termo De Consentimento Livre Esclarecido (TCLE) e descritivo do estudo a ser realizado, elaborado de acordo com a "Declaração de Helsinque III", capítulo 50, parágrafos 50.20/27, que trata da proteção dos participantes e orienta procedimentos referentes às pesquisas que necessitam de experiências com humanos. Em seguida, foi preenchida a ficha de cadastro, após receberem informações detalhadas sobre a pesquisa, com uso de linguagem adequada, conforme estabelecido pela Resolução 466/12 do Conselho Nacional de Saúde (Brasil, 2012).

Após a assinatura do termo, foi realizado o cadastro das participantes e entregue o formulário para preenchimento do registro alimentar, bem como foram agendadas datas para obtenção das medidas antropométricas (peso corporal e estatura), coleta de sangue e entrega do registro alimentar.

\subsection{Avaliação Antropométrica}

Para avaliar o estado nutricional, foi aferido o peso corporal e estatura, conforme descrito pelo Ministério da Saúde (Brasil, 2011). Os dados antropométricos e demais informações das participantes foram anotados na ficha de cadastro das participantes da pesquisa.

\subsubsection{Peso corporal (kg) e Estatura (cm)}

O peso corporal foi determinado utilizando uma balança digital (Plenna ® modelo SIM09190), com capacidade máxima de $180 \mathrm{~kg}$, graduada em 100 gramas, estando as participantes descalças e usando roupas leves. A estatura foi mensurada com antropômetro marca Secar®, graduado em centímetros e com barra vertical e fixa, para o posicionamento da 
cabeça, estando as participantes descalças com os pés unidos, em posição ereta, olhando para frente (Brasil 2011).

O índice de massa corporal (IMC) foi calculado a partir do peso corporal do estudo dividido por sua estatura ao quadrado (WHO, 2008).

$$
\operatorname{IMC}\left(\mathrm{kg} / \mathrm{m}^{2}\right)=(\text { Peso }(\mathrm{kg})) /\left(\text { Altura }(\mathrm{cm})^{2}\right)
$$

A classificação do estado nutricional a partir da distribuição do índice de massa corporal foi realizada segundo a recomendação da World Health Organization (WHO, 2008).

\subsection{Avaliação do consumo alimentar}

Para a avaliação do consumo alimentar, foi utilizado inquérito alimentar realizado de acordo com a técnica de registro alimentar de 3 dias, compreendendo dois dias alternados durante a semana e um dia no final de semana (sábado ou domingo). No momento da entrega dos formulários, as participantes foram orientadas quanto à forma correta de anotar os alimentos, e de discriminar os tipos de refeições, preparações, porcionamento, medidas caseiras, quantidades e horários em que as mesmas foram consumidas. As quantidades de energia, macronutrientes e selênio foram calculadas por meio do programa Nutwin, versão 1.5 do Departamento de Informática em Saúde da Universidade Federal de São Paulo (Anção et al.,2002).

As informações nutricionais dos alimentos e preparações consumidos pelas participantes foram adicionadas ao banco de dados do programa. Para energia e macronutrientes considerou-se a Tabela Brasileira de Composição de Alimentos (TACO, 2011) e a Tabela de Composição de Alimentos do Instituto Brasileiro de Geografia e Estatística 21. Para a inserção das quantidades de selênio, utilizou-se os dados do estudo de Ferreira et al. (2002), da Tabela de Composição de Alimentos do Instituto Brasileiro de Geografia e Estatística (IBGE, 2011a) e do National Nutrient Database for Standard Reference do Departamento de Agricultura dos Estados Unidos (USDA, 2015). As medidas caseiras foram convertidas em gramas utilizando os valores de correspondência referenciados nas tabelas de Pinheiro et al. (2009), IBGE (2011b) e Alvarenga; Alencar; Melo (2005).

Para verificar a adequação da ingestão dietética dos macronutrientes e do selênio, foram utilizados como valores de referência, respectivamente, o intervalo aceitável de distribuição (AMDR) e a necessidade média estimada (EAR), contidos nas Dietary Reference Intakes (DRI's) (IOM, 2006).

\subsection{Coleta do material biológico}

\subsubsection{Coleta de sangue}

A coleta de sangue foi realizada por profissional técnico em enfermagem no período da manhã, entre 7 e 9 horas, estando as participantes em jejum de cerca de 12 horas. Foram coletados $8 \mathrm{~mL}$ de sangue venoso, os quais foram distribuídos em tubos distintos da seguinte forma: (1) tubo a vácuo contendo ácido etileno diaminotetracético (EDTA) para análise do selênio (4 mL de sangue) e (2) tubo vacuette ${ }^{\circledR}$ com ativador de coágulo para a determinação do perfil lipídico (4 mL).

\subsection{Determinação dos Parâmetros Bioquímicos}

\subsubsection{Controle de Contaminação e Preparo de Reagentes}

A fim de garantir o controle de contaminação por minerais, toda a vidraria e material de polipropileno utilizado para as análises foram desmineralizados antes do uso, por meio do banho em solução de ácido nítrico a 30\%, durante um período mínimo de 12 horas. Posteriormente, foram enxaguados em água deionizada, no mínimo 10 vezes, secos em estufa e mantidos em depósitos fechados, até o momento da utilização (Instituto Adolfo Lutz, 1985).

Todos os reagentes utilizados foram de grau de pureza analítica (P.A). Todas as soluções aquosas e as diluições foram 


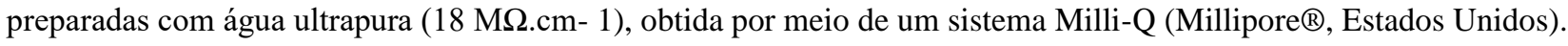

\subsubsection{Separação dos Componentes do Sangue}

Para determinação do selênio nos eritrócitos, a massa eritrocitária foi lavada com 3,5 mL de solução salina isotônica ( $\mathrm{NaCl}$ a $0,9 \%)$, sendo cuidadosamente homogeneizada por inversão e, posteriormente, centrifugada a $2401 \mathrm{xg}$ por 10 minutos. Este procedimento foi repetido três vezes para remover contaminantes dos eritrócitos (plaquetas e leucócitos). Após a última centrifugação, a solução salina foi aspirada e descartada, e a massa eritrocitária foi extraída cuidadosamente com o auxílio de uma pipeta automática e transferida para microtubos desmineralizados, que foram mantidos à temperatura de $-35^{\circ} \mathrm{C}$ para posterior análise (Whitehouse et al.,1982).

\subsubsection{Determinação do selênio eritrocitário}

A análise do selênio foi realizada no Laboratório de Espectrometria de Emissão Atômica da Empresa Brasileira de Pesquisa Agropecuária (Embrapa - Centro Nacional de Pesquisa de Milho e Sorgo), em Belo Horizonte, Minas Gerais.

As amostras de eritrócitos/papa de hemácias foram diluídas em 1:60 (v/v) da seguinte forma: 3,0\% (m/v) 1-butanol, $0,2 \%$ (v/v) TAMA (surfactante de alta pureza), $0,1 \%$ (v/v) HNO3.

O Se+6 foi reduzido para $\mathrm{Se}+4 \mathrm{em} \mathrm{HCl} 4 \mathrm{M}$, esquentando a solução diluída a $90^{\circ} \mathrm{C}$ por $30 \mathrm{~min}$. A escolha das linhas espectrais de análise foi baseada tanto na sua sensibilidade quanto na interferência espectral, sendo otimizadas para o selênio leituras nos comprimentos de onda de 196,026 nm e 203,985 nm. As amostras foram medidas em triplicata.

Solução estoque de $1000 \mu \mathrm{g} / \mathrm{mL}$ de selênio (Spex®CertiPrep, Estados Unidos) foi utilizada para preparar as curvas de calibração e otimização das condições analíticas. Todos os padrões foram preparados da mesma forma que as amostras. As curvas de calibração foram preparadas nas seguintes concentrações: 1, 5, 10, 20, 50 e $100 \mu \mathrm{g} / \mathrm{L}$ em soluções diluentes contendo 3,0\% (m/v) 1-butanol, 0,1\% (v/v) TAMA (surfactante de alta pureza) e 0,05\% (v/v) HNO3. Os limites de detecção foram determinados a partir da equação: $3 \times$ desvio padrão de 10 medições do branco, dividido pela declividade da curva de calibração. Amostras de material de referência certificado (Seronorm® Oligoelement Serum, Noruega) foram determinadas para validar as medições analíticas em ICP-OES.

Para avaliação do estado nutricional relativo ao selênio foram adotados os seguintes valores de referência: selênio

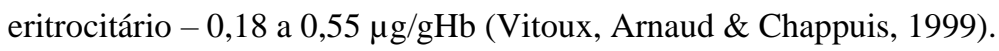

\subsubsection{Determinação de hemoglobina}

A concentração de hemoglobina na massa eritrocitária foi determinada conforme o método da cianometaemoglobina para expressar as concentrações de selênio eritrocitário (Van Assendelft, 1972). Esse método se baseia na reação de oxidação do $\mathrm{Fe}+2$ do grupo heme da hemoglobina pelo ferricianeto, e posterior reação com o cianeto de potássio para formar cianeto de hemoglobina.

Inicialmente, em um microtubo contendo $300 \mu \mathrm{L}$ de massa eritrocitária foi adicionado $900 \mu \mathrm{L}$ de água ultrapura, perfazendo um lisado com proporção 1:4. Em seguida, a uma alíquota de $20 \mu \mathrm{L}$ desse lisado foi acrescentado $5 \mathrm{~mL}$ de solução de Drabkin (Labtest ${ }^{\circledR}$, Brasil). A leitura da absorbância foi realizada em espectrofotômetro UV visível (Bel Photonics®, SP1102, Brasil) considerando o comprimento de onda de $540 \mathrm{~nm}$.

\subsubsection{Determinação dos lipídios séricos}

As concentrações séricas de colesterol total, HDL-colesterol e triacilgliceróis foram determinadas segundo método enzimático colorimétrico, por analisador bioquímico automático COBAS INTEGRA (Roche Diagnostics, Brasil), utilizando 
kits ROCHE®. A fração LDL-colesterol foi calculada de acordo com a fórmula de Friedwald, Levy \& Fredrickson (1972): LDL-c $=$ CT - HDL-c $-\mathrm{TG} / 5$, sendo válida para valores de triglicérides até $400 \mathrm{mg} / \mathrm{dL}$. A fração de VLDL-colesterol foi calculada segundo a fórmula: $\mathrm{VLDL}=\mathrm{TG} / 5$.

Os valores para lipídios séricos, definidos na Atualização da Diretriz Brasileira de Dislipidemias e Prevenção da Aterosclerose - 2017 foram utilizados como padrão de referência (Faludi et al., 2017).

\subsection{Análise Estatística}

Os dados foram organizados em planilhas do Microsoft Excel @ para realização da análise descritiva das variáveis observadas. Posteriormente, os dados foram exportados para o programa SPSS (for Windows® versão 20.0) para análise das estatísticas dos resultados.

Os testes de Kolmogorov-Smirnov e de Shapiro-Wilk foram aplicados para verificar a homocedasticidade dos dados. Em seguida, para fins de comparação entre os grupos, o teste $t$ de Student foi utilizada para as variáveis com distribuição normal, e o teste de Mann Whitney para aquelas com distribuição não paramétrica. Nas análises de comparação entre mais de dois grupos foi realizada a análise de variância de uma via (ANOVA one-way), adotando-se o teste post-hoc de Tukey.

Para o estudo de correlações, o coeficiente de correlação linear de Pearson foi utilizado para os dados com distribuição normal, e o coeficiente de correlação de postos de Spearman para aqueles com distribuição não paramétrica. A diferença foi considerada estatisticamente significativa quando o valor de $\mathrm{p}<0,05$, admitindo-se um erro de $5 \%$.

\section{Resultados}

\subsection{Parâmetros Antropométricos De Avaliação Do Estado Nutricional}

Os parâmetros antropométricos estão descritos na Tabela 1.

Tabela 1. Valores médios e desvios padrão da idade, peso corporal, estatura, circunferência da cintura e índice de massa corpórea das mulheres obesas e grupo controle. Teresina-PI, Brasil, 2021.

\begin{tabular}{cccc}
\hline Parâmetros & $\begin{array}{c}\text { Caso }(\mathbf{n}=\mathbf{2 2}) \\
\text { Média } \pm \mathbf{D P}\end{array}$ & $\begin{array}{c}\text { Controle }(\mathbf{n}=\mathbf{3 4}) \\
\text { Média } \pm \mathbf{D P}\end{array}$ & $\mathbf{p}$ \\
\hline Idade $(\mathbf{a n o s})^{\mathbf{a}}$ & $34,04 \pm 8,64$ & $34,71 \pm 7,46$ & 0,762 \\
\hline Peso corporal $(\mathbf{k g})^{\mathbf{b}}$ & $109,66 \pm 15,25^{*}$ & $55,81 \pm 5,08$ & $<0,001$ \\
\hline Estatura $(\mathbf{m})^{\mathbf{a}}$ & $1,61 \pm 0,05^{*}$ & $1,58 \pm 0,07$ & 0,034 \\
\hline IMC $\left(\mathbf{k g} / \mathbf{m}^{\mathbf{2}}\right)^{\mathbf{b}}$ & $42,26 \pm 6,78^{*}$ & $22,35 \pm 1,58$ & $<0,001$ \\
\hline
\end{tabular}

Fonte: Dados da pesquisa. *Valores significativamente diferentes entre as pacientes obesas e grupo controle, teste $t$ de Student $t^{\text {a }}$ ou teste Mann-Whitney ${ }^{\mathrm{b}}(\mathrm{p}<0,05)$. IMC = Índice de Massa Corporal.

Os valores médios e desvios padrão da idade e dos parâmetros antropométricos utilizados na avaliação do estado nutricional das participantes deste estudo estão apresentados na Tabela 1. Observou-se diferença estatística significativa para os parâmetros peso, estatura, circunferência da cintura e índice de massa corporal $(p<0,05)$, sendo superiores nas mulheres obesas.

\subsection{Consumo Alimentar}

A quantidade de ingestão de energia, macronutrientes e selênio ingerida pelas mulheres encontra-se na Tabela 2. 
Tabela 2. Valores médios e desvios padrão da ingestão de energia, macronutrientes e selênio das mulheres obesas e grupo controle. Teresina-PI, Brasil, 2021.

\begin{tabular}{|c|c|c|c|}
\hline Energia/Nutrientes & $\begin{array}{l}\text { Caso }(n=22) \\
\text { Média } \pm \text { DP }\end{array}$ & $\begin{array}{c}\text { Controle }(n=34) \\
\text { Média } \pm \text { DP }\end{array}$ & $\mathbf{p}$ \\
\hline Energia $\left(\right.$ Kcal) ${ }^{b}$ & $1704,47 \pm 329,46$ & $1668,49 \pm 404,29$ & 0,568 \\
\hline Carboidrato $(\%)^{\mathbf{b}}$ & $51,36 \pm 5,98$ & $50,70 \pm 7,12$ & 0,907 \\
\hline Proteína $(\%)^{\mathrm{a}}$ & $19,94 \pm 3,71$ & $19,91 \pm 3,44$ & 0,975 \\
\hline Lipídio $(\%)^{\mathrm{b}}$ & $28,70 \pm 3,76$ & $29,39 \pm 5,68$ & 0,513 \\
\hline Selênio dietético $(\mu \mathrm{g})^{\mathrm{b}}$ & $78,64 \pm 21,35$ & $70,28 \pm 26,29$ & 0,107 \\
\hline
\end{tabular}

Fonte: Dados da pesquisa. Teste $t$ de Student $t^{\mathrm{a}}$ ou teste Mann-Whitney ${ }^{\mathrm{b}}(\mathrm{p}<0,05)$. Valores de referência: 10 a $35 \%$ de proteína, 20 a $35 \%$ de lipídio, 45 a $65 \%$ de carboidratos e $55 \mu \mathrm{g}$ de selênio (INSTITUTE OF MEDICINE, 2005).

Os valores médios e desvios padrão da ingestão de energia, macronutrientes e selênio encontrados nas dietas consumidas pelas participantes do estudo estão descritos na Tabela 2. Não foi verificada diferença estatística significativa entre as mulheres obesas e grupo controle $(\mathrm{p}>0,05)$.

\subsection{Parâmetros Bioquímicos de Avaliação do Selênio}

A concentração eritrocitária de selênio encontrada nas mulheres obesas e grupo controle está apresentada na Tabela 3.

Tabela 3. Valores médios e desvios-padrões das concentrações eritrocitárias de selênio das mulheres obesas e grupo controle. Teresina-PI, Brasil, 2021

\begin{tabular}{|c|c|c|c|}
\hline \multirow{2}{*}{ Parâmetros } & Caso $(n=22)$ & Controle $(n=34)$ & \multirow{2}{*}{$\mathbf{p}$} \\
\hline & Média \pm DP & Média \pm DP & \\
\hline Selênio eritrocitário ${ }^{a}$ & $0,25 \pm 0,03^{*}$ & $0,39 \pm 0,08$ & $<0,001$ \\
\hline
\end{tabular}

Fonte: Dados da pesquisa. *Valores significativamente diferentes entre as pacientes obesas e grupo controle, teste $t$ de Student ${ }^{\text {a }}$ ou teste Mann-Whitney ${ }^{\mathrm{b}}(\mathrm{p}<0,05)$. Valores de referência: 0,18 a $0,55 \mu \mathrm{g} / \mathrm{gHb}$ de selênio eritrocitário.

Na Tabela 3 encontram-se as concentrações de selênio eritrócito das participantes obesas e grupo controle. As mulheres obesas apresentaram valores inferiores de selênio eritrócito, quando comparadas ao grupo controle, com diferença estatística significativa $(\mathrm{p}<0,05)$.

\subsection{Determinação Do Perfil Lipídico}

O perfil lipídico das mulheres obesas e grupo controle estão apresentadas na Tabela 4. 
Tabela 4. Valores médios e desvios padrão dos parâmetros do perfil lipídico das mulheres obesas e grupo controle. TeresinaPI, Brasil, 2021.

\begin{tabular}{cccc}
\hline Parâmetros & $\begin{array}{c}\text { Caso }(\mathbf{n}=\mathbf{2 2}) \\
\text { Média } \pm \text { DP }\end{array}$ & $\begin{array}{c}\text { Controle }(\mathbf{n}=\mathbf{3 4}) \\
\text { Média } \pm \text { DP }\end{array}$ & $\mathbf{p}$ \\
\hline Colesterol total & $193,64 \pm 24,37^{*}$ & $173,18 \pm 20,85$ & 0,001 \\
\hline Triacilgliceróis & $141,54 \pm 50,16^{*}$ & $98,26 \pm 27,39$ & 0,001 \\
\hline VLDL-c & $28,31 \pm 10,03^{*}$ & $19,65 \pm 5,48$ & 0,001 \\
\hline HDL-c & $48,32 \pm 12,41^{*}$ & $55,06 \pm 9,27$ & 0,024 \\
\hline LDL-c & $117,01 \pm 23,87^{*}$ & $98,46 \pm 20,14$ & 0,003
\end{tabular}

Fonte: Dados da pesquisa. *Valores significativamente diferentes entre as pacientes obesas e grupo controle, teste $t$ de $S t u d e n t(p<0,05)$. VLDL-c = lipoproteína de muito baixa densidade; HDL-c = lipoproteína de alta densidade; LDL-c = lipoproteína de baixa densidade. Metas para o perfil lipídico em jejum: Colesterol total <190, HDL >40, triacilgliceróis <150, LDL <130.

Na Tabela 4 encontram-se os valores médios e desvios padrão dos parâmetros do perfil lipídico das participantes obesas e grupo controle. As mulheres obesas apresentaram valores superiores de colesterol total, triacilgliceróis, VLDL-c e LDL-c, e valores inferiores de HDL-c quando comparados ao grupo controle, com diferença significativa $(\mathrm{p}<0,05)$.

\subsection{Correlação entre os Biomarcadores do Selênio e Parâmetros do Perfil Lipídico}

Os resultados da análise de correlação entre os biomarcadores do selênio e parâmetros do perfil lipídico no grupo das mulheres obesas encontram-se na Tabela 5.

Tabela 5. Análise de correlação linear simples entre biomarcadores do selênio e parâmetros do perfil lipídico nas mulheres obesas. Teresina-PI, Brasil, 2021.

\begin{tabular}{ccccc}
\hline \multirow{2}{*}{ Parâmetros } & \multicolumn{2}{c}{ Selênio dietético } & \multicolumn{2}{c}{ Selênio eritrocitário } \\
\cline { 2 - 5 } & $\mathbf{r}$ & $\mathbf{p}$ & $\mathbf{r}$ & $\mathbf{p}$ \\
\hline CT & $-0,267$ & 0,229 & 0,270 & 0,223 \\
\hline VL & $-0,208$ & 0,353 & 0,116 & 0,607 \\
\hline HDL-c & $-0,208$ & 0,353 & 0,116 & 0,607 \\
\hline LDL-c & 0,110 & 0,625 & 0,267 & 0,230 \\
\hline
\end{tabular}

Fonte: Dados da pesquisa. *Correlação Linear de Pearson $(\mathrm{p}<0,05) . \mathrm{CT}=$ colesterol total; TG = triacilgliceróis; HDL-c = lipoproteína de alta densidade; LDL-c = lipoproteína de baixa densidade; VLDL-c = lipoproteína de muito baixa densidade.

Os resultados da análise de correlação entre os biomarcadores do selênio e parâmetros do perfil lipídico no grupo controle encontram-se na Tabela 6. 
Tabela 6. Análise de correlação linear simples entre biomarcadores do selênio e parâmetros do perfil lipídico no grupo controle. Teresina-PI, Brasil, 2021.

\begin{tabular}{ccccc}
\hline \multirow{2}{*}{ Parâmetros } & \multicolumn{2}{c}{ Selênio dietético } & \multicolumn{2}{c}{ Selênio eritrocitário } \\
\cline { 2 - 5 } & $\mathbf{r}$ & $\mathbf{P}$ & $\mathbf{r}$ & $\mathbf{P}$ \\
\hline CT & $-0,010$ & 0,954 & 0,071 & 0,689 \\
\hline TG & 0,116 & 0,513 & $-0,133$ & 0,454 \\
\hline VLDL-c & 0,116 & 0,513 & $-0,133$ & 0,454 \\
\hline HDL-c & 0,100 & 0,575 & $-0,132$ & 0,458 \\
\hline LDL-c & $-0,104$ & 0,557 & 0,170 & 0,336 \\
\hline
\end{tabular}

Fonte: Dados da pesquisa. *Correlação Linear de Pearson $(\mathrm{p}<0,05) . \mathrm{CT}=$ colesterol total; TG = triacilgliceróis; HDL-c = lipoproteína de alta densidade; LDL-c = lipoproteína de baixa densidade; VLDL-c = lipoproteína de muito baixa densidade.

As Tabelas 5 e 6 apresentas a análise de correlação entre os biomarcadores de selênio e os parâmetros do perfil lipídico, em que não se observou correlação significativa entre o selênio dietético e eritrocitário e os parâmetros do perfil lipídico nos grupos avaliados.

\section{Discussão}

Neste estudo foram avaliados biomarcadores do selênio em mulheres obesas, bem como foi investigada a existência de correlação entre essas variáveis e parâmetros do perfil lipídico. Em relação ao consumo alimentar, observou-se que a ingestão de energia e de macronutrientes não apresentou diferença estatística significativa entre os grupos caso e controle. Os resultados encontrados mostram consumo habitual dos macronutrientes dentro dos valores recomendados pelas DRIs, em ambos os grupos, semelhante ao observado por Pereira et al. (2012), Sousa et al. (2019) e Sousa et al. (2019).

Sobre tal resultado, destaca-se que o consumo alimentar semelhante entre os grupos pode ser justificado pelo fato de os indivíduos obesos frequentemente subestimarem a ingestão dietética, principalmente de alimentos ricos em açúcar e gordura (Mayer et al., 2018). No entanto, o registro alimentar, instrumento utilizado para estimar o consumo dietético, consiste em um método validado para identificar o consumo habitual de alimentos (Shim, Oh \& Kim, 2014).

As quantidades de selênio presentes nas dietas consumidas pelas mulheres com obesidade estavam adequadas, segundo as recomendações nutricionais constantes nas DRI's. No entanto, é importante enfatizar que o teor de selênio nos alimentos pode sofrer variação por diversos fatores, dentre eles as alterações geográficas desse mineral no solo, a forma de preparação dos alimentos e as interações com outros nutrientes, o que pode interferir na sua biodisponibilidade. Além disso, a necessidade do uso de tabelas estrangeiras e a falta de distinção da quantidade desse mineral em alimentos das diferentes regiões geográficas do Brasil podem superestimar a sua ingestão (Fairweather-Tait, Collings \& Hurst, 2011; Cozzolino, 2012; Mehdi et al., 2013).

Nesse sentido, tem sido verificado na comunidade científica interesse para alterar as quantidades recomendadas de ingestão de selênio, em particular para indivíduos com obesidade. A sugestão é que o teor seja superior aos valores já estabelecidos, em torno de $1 \mu \mathrm{g} / \mathrm{kg} / \mathrm{dia}$ para manter o estado nutricional adequado do nutriente no organismo (DammsMachado, Weber \& Bischoff, 2012; Kipp et al., 2015).

$\mathrm{O}$ estudo mostrou menor concentração de selênio eritrocitário nas mulheres com obesidade quando comparado ao grupo controle, sendo o estresse oxidativo um possível fator contribuinte para tal resultado, pois o aumento de espécies reativas de oxigênio presente na obesidade favorece a redução das concentrações plasmáticas de cofatores de enzimas antioxidantes, a 
exemplo do selênio (Tascilar et al., 2011; Murer et al., 2013; Patel, Kishore \& Patel, 2014). Além disso, verifica-se elevada demanda desse nutriente para a síntese de selenoproteínas antioxidantes em alguns tecidos, fato que também pode ter contribuído para a sua redução nos eritrócitos (Liao et al., 2018).

Outro ponto importante a ser mencionado, diz respeito ao papel da inflamação crônica de baixo grau presente na obesidade, a qual também pode ter favorecido a redução da concentração eritrocitária de selênio, em vista da provável redução na expressão de selenoproteína P, proteína importante na regulação da concentração de selênio (Zhong, Lin \& Nong, 2018).

Nessa discussão, destaca-se que embora as concentrações de selênio eritrocitário estivessem inferiores nas mulheres com obesidade, as mesmas se encontravam dentro do intervalo de referência. Esse resultado pode ser explicado provavelmente pela meia vida longa de 120 dias dos eritrócitos e o turnover lento de selênio nessas células, tornando-o menos responsivo à sua deficiência (Franco, 2012).

No que diz respeito à avaliação dos parâmetros do perfil lipídico, os resultados mostraram diferença estatística significativa nas variáveis: colesterol total, triacilgliceróis, LDL-c, VLDL-c e HDL-c, entre os grupos avaliados. Corroborando com resultados obtidos por Kanwar e Kabra (2016) e Khan e Khaleel (2016). Esses dados reforçam a existência de uma associação entre a presença da obesidade e o aumento do risco de desordens cardiovasculares, a exemplo das dislipidemias (Vekic et al., 2018).

Na perspectiva de uma melhor compreensão do papel do selênio sobre o perfil lipídico, conduziu-se análise de correlação entre o teor dietético e a concentração eritrocitária desse mineral e parâmetros do perfil lipídico, que não evidenciou resultado significativo. Possivelmente o número amostral reduzido, a técnica de avaliação do consumo alimentar, que apesar de ser o método mais utilizado em pesquisas, apresenta vieses inerentes, como a subestimação no consumo de alguns alimentos podem ser fatores que contribuíram para este resultado.

Diante do exposto, considerando a complexidade dos mecanismos envolvidos na participação do selênio no controle da dislipidemia em pacientes obesos, é importante investigar a atuação do mineral em distúrbios lipídicos para um melhor entendimento acerca do comportamento metabólico desse nutriente na patogênese da obesidade.

\section{Conclusão}

A partir dos resultados do estudo, pode-se concluir que as mulheres obesas ingerem quantidade adequada de selênio, mas possuem concentrações eritrocitárias reduzidas e apresentam alterações nos parâmetros do perfil lipídico. Além disso, o estudo não revela correlação entre o marcador dietético e eritrocitário de selênio e os parâmetros do perfil lipídico.

Considerando que as mulheres obesas avaliadas apresentam ingestão de selênio adequada, mas as concentrações do mineral nos eritrócitos encontram-se reduzidas, entende-se que existe a necessidade de mais estudos dessa natureza para contribuir na elucidação sobre a distribuição do selênio em organismos com obesidade. Além disso, é relevante a realização de estudos que esclareçam o papel desse elemento na prevenção da dislipidemia e, consequentemente no desenvolvimento de doenças cardiovasculares.

\section{Referências}

Alasfar, F. et al. (2011). Selenium Is Significantly Depleted Among Morbidly Obese Female Patients Seeking Bariatric Surgery. Obesity Surgery, 21(11), $1710-1713$.

Alvarenga, E. S. L., Alencar, M. A. P. M. \& Melo, M. T. S. M. (2005). Alimentos - Medidas caseiras: correspondência peso-volume. Teresina: UFPI.

Anção, M. S. et al. (2002). Programa de apoio à nutrição Nutwin: versão 1.5. Departamento de Informática em Saúde [CD-ROM] Unifesp/EPM.

Bleys, J. et al. (2008). Serum selenium and serum lipids in US adults. The American Journal of Clinical Nutrition, 88(2), 416-423. 
Brasil. (2012). Ministério da Saúde. Conselho Nacional de Pesquisa com Seres Humanos. Resolução n466, de 12 de dezembro de 2012. Diário Oficial da União, Brasília, DF.

Brasil. (2011). Ministério da Saúde. Secretaria de Atenção à Saúde. Departamento de Atenção Básica. Orientações para a coleta e análise de dados antropométricos em serviços de saúde: Norma Técnica do Sistema de Vigilância Alimentar e Nutricional - SISVAN. Brasília: Ministério da Saúde.

Choe, S. S. et al. (2016). Adipose Tissue Remodeling: Its Role in Energy Metabolism and Metabolic Disorders. Frontiers in Endocrinology, 7(1), 1-6.

Cozzolino, S. M. F. (2012). Biodisponibilidade de nutrientes. (4a ed.), Manole.

Damms-Machado, A., Weber, G. \& Bischoff, S. C. (2012). Micronutrient Deficiency in Obese Subjects Undergoing Low Calorie Diet. Nutrition Journal, 11(1), 1-8.

Dhingra, S. \& Bansal, M. P. (2006). Modulation of hypercholesterolemia-induced alterations in apolipoprotein B and HMG-CoA reductase expression by selenium supplementation. Chemico-Biological Interactions, 161, 49-56.

Fairweather-Tait, S. J., Collings, R. \& Hurst, R. (2011). Selenium in human health and disease. Antioxidants \& Redox Signaling, 14(7), $1337-1383$.

Faludi, A. A. et al. (2017). Atualização da Diretriz Brasileira de Dislipidemias e Prevenção da Aterosclerose - 2017. Arquivos Brasileiros de Cardiologia, 109 (2, Suppl. 1), 1-76.

Ferreira, K. S. et al. (2002). Concentrações de selênio em alimentos consumidos no Brasil. Pan Am J Public Health, 11(3), 172-177.

Franco, R. S. (2012). Measurement of red cell lifespan and aging. Transfusion Medicine and Hemoterapy, 39(5), 302-207.

Friedewald, W.T., Levy, R.I. \& Fredrickson, D.S. (1972). Estimation of the concentration of low-density lipoprotein cholesterol in plasma, without use of the preparative ultracentrifuge. Clinical Chemistry, 18(6), 499-502.

Fontenelle, L. C. et al. (2018). The role of selenium in insulin resistance. Brazilian Journal of Pharmaceutical Sciences, 54(1), 1-11.

Fülöp, P. et al. (2013). Increased hair selenium concentration in hyperlipidemic patients. Journal of Cellular and Molecular Medicine, 17(3), 350-355.

IBGE (Instituto Brasileiro de Geografia e Estatística) (2011a). Pesquisa de Orçamentos Familiares 2008-2009: tabelas de composição nutricional dos alimentos consumidos no Brasil. Rio de Janeiro: IBGE.

IBGE (Instituto Brasileiro de Geografia e Estatística) (2011b). Pesquisa de Orçamentos Familiares 2008-2009: tabela de medidas referidas para os alimentos consumidos no Brasil. Rio de Janeiro.

IOM (Institute of Medicine) (2006). Dietary reference intakes: the essential guide to nutrient requirements. National Academy Press.

Instituto Adolfo Lutz. (1985). Normas analíticas do Instituto Adolfo Lutz. (3a ed.), 1,1-533.

Ju, W. et al. (2017). Relationship between higher serum selenium level and adverse blood lipid profile. Clinical Nutrition, 37(5), 1-6.

Kanwar, G. \& Kabra, R. (2016). A study of association between obesity and lipid profile. Impact Journals, 4(4), 69-74.

Khan, M. N. \& Khaleel, M. (2016). Comparative study of sérum lipid profile of obese and non-obese students (male) of Aljouf University. International Journal of Biomedical and Advance Research, 7(1), 35-37.

Kipp, A. P. et al. (2015). Revised reference values for selenium intake. Journal of Trace Elements in Medicine and Biology, 32(1), 195-199.

Liao, C. et al. (2018). The Intricate Role of Selenium and Selenoproteins in Erythropoiesis. Free Radical Biology and Medicine, 127(1), 165-171.

Mayer, D. et al. (2018). Dieta hiperlipídica e excesso de gordura corporal em colabores de uma Hospital Universitário em Curitiba-PR. Revista Brasileira de Obesidade, Nutrição e Emagrecimento, 12 (74), 722-729.

Mehdi, Y. et al. (2013). Selenium in the Environment, Metabolism and Involvement in Body Functions. Molecules, 18(3), 3292-3311.

Murer, S. B. et al. (2013) Antioxidant supplements reduced oxidative stress and stabilized liver function tests but did not reduce inflammation in a randomized controlled trial in obese children and adolescents. Journal of Nutrition, 144(2), 193-201.

Patel, M. D., Kishore, P. K. \& Patel, D. J. (2014). Evaluation of oxidative stress and serum magnesium levels in South Indian obese males. International Journal of Scientific Research, 3(3), 229-230.

Petry, E. R. et al. Suplementações Nutricionais e Estresse Oxidativo. Revista Brasileira de Ciência e Esporte, 35(4), 1071-1092.

Pereira, A.S et al. (2018). Metodologia da pesquisa científica. UFSM. https://repositorio.ufsm.br/bitstream/handle/1/15824/Lic_Computacao_MetodologiaPesquisa-Cientifica.pdf?sequence $=1$.

Pereira, L. S. S. et al. (2012). Relação Entre Perfil Antropométrico E A Ingestão Dietética Em Pacientes Atendidos Na Clínica Escola Da Faculdade Pitágoras, Campus Ipatinga-Mg. Revista Brasileira de Obesidade, Nutrição e Emagrecimento, 6(31), 4-12.

Stranges, S. et al. (2010). Higher selenium status is associated with adverse blood lipid profile in British adults. Journal of Nutrition, 140, 81-87.

Sousa, M. P. et al. (2019). Associação entre Ingestão Dietética de Magnésio e Parâmetros do Perfil Lipídico em Mulheres obesas. Research, Society and Development, 9(1) 1 - 17 . 
Research, Society and Development, v. 10, n. 3, e36410313476, 2021

(CC BY 4.0) | ISSN 2525-3409 | DOI: http://dx.doi.org/10.33448/rsd-v10i3.13476

Sousa, T. G. V. et al. (2019). Ingestão de magnésio e ferro com estresse oxidativo em mulheres obesas. Research, Society and Development, 9(1), 1 - 17.

Shim, J., Oh, K. \& Kim, H. C. (2014). Dietary assessment methods in epidemiologic studies. Epidemiology and Health, 36, 1-8.

Su, L. et al. (2015). Selenium Level and Dyslipidemia in Rural Elderly Chinese. PLoS One, 10(9), 1-11.

TACO. (2011). Tabela Brasileira de Composição de Alimentos. (4a ed.), NEPA-UNICAMP.

Tangvarasittichai, S. (2015). Oxidative stress, insulin resistance, dyslipidemia and type 2. World Journal of Diabetes, 6(3), 456 - 480.

Tascilar, M. E. et al. (2011). Trace elements in obese turkish children. Biological Trace Elements Research, 143, 188-195.

Uranga, R. M. \& Keller, J. N. (2019). The Complex Interactions Between Obesity, Metabolism and the Brain. Frontiers in Neuroscience, $13(1), 1-21$.

USDA (Department of Agriculture). (2015). National Nutrient Database for Standard Reference: selenium (release 28).

Van Assendelft, O. W. (1972). The measurement of hemoglobin. In: Izak, G. \& Lewis, S. M. Modern concepts in hematology: symposia of the International Committee for Standardization in Hematology. New York: Academic Press, 14-25.

Vitoux, D., Arnaud, J. \& Chappuis, P. (1999). Are copper, zinc and selenium in erythrocytes valuable biological indexes of nutrition and pathology? Journal of Trace Elements in Medicine and Biology, 13(3), 113-128.

Vekic, J. et al. (2018). Obesity and dyslipidemia. Metabolism, 92, 71-81.

WHO (World Health Organization). (2008). Obesity: preventing and managing the global epidemic - report of a WHO consultation. Geneva: World Health Organization.

Whitehouse, R. C. et al. (1982). Zinc in plasma, neutrophils, lymphocytes, and erythrocytes as determined by flameless atomic absorption spectrophotometry. Clinical Chemistry, 28(3), 475-480.

Yang, K. C. et al. (2010). Serum selenium concentration is associated with metabolic factors in the elderly: a cross-sectional study. Nutrition \& Metabolism, $7(38)$.

Xavier, H. T. et al. (2013). V Diretriz Brasileira de Dislipidemias e Prevenção da Aterosclerose. Arquivo Brasileiro Cardiologia, 101(4), 1-20.

Zhong, Q., Lin, R. \& Nong, Q. (2018). Adiposity and serum selenium in U.S adults. Nutrients, 10(727), 1-11. 